\title{
Preparation of Amazonian Palm Tree Fiber (Manicaria saccifera Gaertn.) for Composite Materials
}

\author{
Amanda Sousa Monteiro ${ }^{1}$, Denise Dantas ${ }^{2}$, Takashi Yojo ${ }^{3}$ \\ ${ }^{1}$ Post Graduation Program in Design, Faculty of Architecture and Urbanism, University of São \\ Paulo, R. do Lago 876, 05508-080 São Paulo, Brazil (amandasousamonteiro@usp.br) ORCID \\ 0000-0002-9965-0390; 'Post Graduation Program in Design, Faculty of Architecture and \\ Urbanism, University of São Paulo, R. do Lago 876, 05508-080 São Paulo, Brazil \\ (dedantas@usp.br) ORCID 0000-0003-4419-6394; 3 ${ }^{2}$ aboratory Trees, Woods and Furniture, \\ IPT - Institute for Technological Research, Av. Prof. Almeida Prado 53, 05508-901 São Paulo, \\ Brazil (yojos@ipt.br) ORCID 0000-0002-0695-6973
}

\begin{abstract}
In the last fifteen years, a new approach has emerged in the design field: the process of development of new materials and the involvement of designers as original creators, making use of underutilized local materials. This paper aims to describe the preparation of an Amazonian palm tree fiber (Manicaria saccifera Gaertn.) for the use at composites materials. The raw materials were extracted from the Marajó archipelago - Brazil by local communities that traditionally work with the material. The process involves the measure of weight, length, and thickness at six different points of the raw specimen; manual washing and cleaning; drying with stopping criterion; and visual analyses of the specimen with trimming and cutting, for the potential use at composites. The results for 80 specimens show that the method adopted allowed easy and quick preparation of the specimens and can be used for other local materials, respecting its characteristics.
\end{abstract}

Author Keywords. Tururi Fiber, Amazonian Fiber, Composite Materials, Preparation of Samples.

Type: Research Article

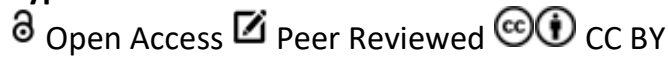

\section{Introduction}

The innumerable changes that occurred in nature and society after the industrial revolution has created the search for more responsible and sustainable alternatives in the field of industrial design. Hence, in contemporary industrialized societies, the relationship between human beings, materials, and technology has become much more complex (Manzini and Vezzoli 2008, 20-23).

According to Manzini and Vezzoli $(2008,71)$, designers can act in this scenario in three ways: increasing the alternatives through strategies aimed at problem-solving; promoting their ability to personally intervene in the direction of results and the means to achieve them; or stimulating their own imagination, envisioning creative solutions to cultural and social problems.

A way to achieve those parameters working from the perspective of creative experimentation is through materials. Turning these data into goals, a new approach has emerged in the design field: the process of development of new materials, and the involvement of designers as original creators (Rognoli et al. 2015, 692; Karana, Hekkert, and Kandachar 2008, 36; AaylaGarcia and Rognoli 2017, 375). The unique creative challenge provided by experimentation with materials generates a language that can be developed into a new design path. 
Understanding the material is the first step to work with them. For Ashby and Johnson (2011, 55-57), the physical parameters need to be well-defined in order to propose new and efficient usages. Such knowledge is a result of characterizations that generate raw data, which need to be distilled by appropriate statistical analysis that can be transformed into attributes known as "material properties". Using such data safely, within the dimension of a product's actual use, is a big challenge.

Part of understanding a material, after appropriate physical and chemical tests, is knowing how it should be treated and processed. Natural materials, specifically, need to be thoroughly analyzed so that the alternatives that arise during a project are likely to be applied (Manzini 1993, 42-45).

This process can lead to materials with great potential for innovation, such as polymeric composites with natural fibers, which allow the union between the development of new materials and creative thinking. Furthermore, it provides the intersection between the areas of design and materials engineering, an interdisciplinary study of great interest, and the growth potential may benefit both areas (Ashby and Johnson 2011, 163-65). Working with materials enables a great number of creative directions, therefore, it is important to analyze what kind of materials are being applied in design projects and what can be done in some specific areas, namely local materials, respecting important points such as the original population that traditionally makes use of it.

In the Brazilian and Amazonian context, there is an incredible amount of natural fibers that are underutilized in the industry. One of those is the "tururi" fiber, which was selected to conduct this work (Monteiro and Baruque-Ramos 2016, 127).

\section{Materials and Methods}

Tururi is a fiber material, derived from the bunches of fruits of the Ubuçu palm (Manicaria saccifera Gaertn) (Figure 1). It is a species native to Central America, Venezuela, Colombia, Guyana, and Brazil. In Brazil, the Ubuçu palm tree comes from the Amazon region and it is found mainly in the states of Amazonas, Pará, and Amapá. In these states, the palm is found abundantly in the floodplain and island forests (Monteiro and Baruque-Ramos 2016, 127).

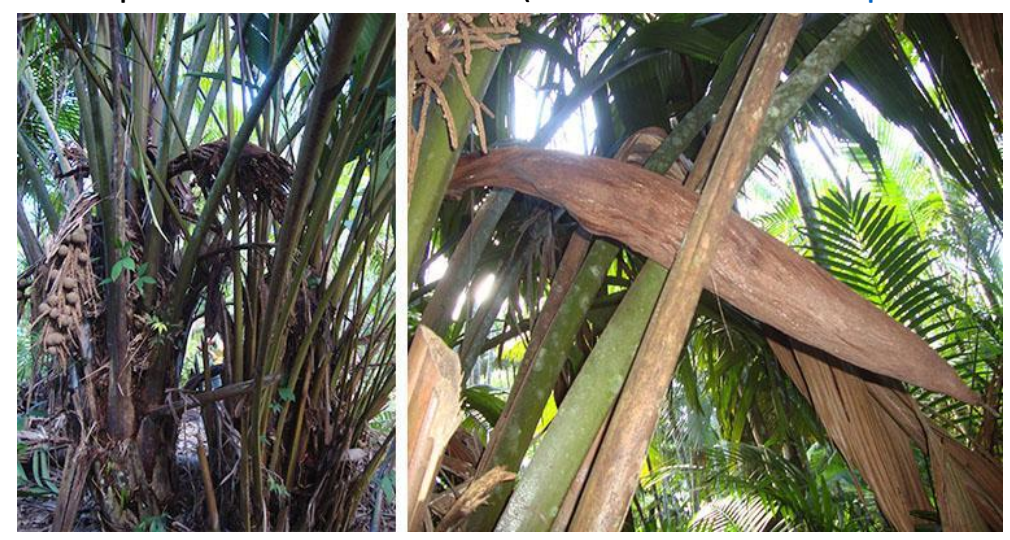

Figure 1: Ubuçu Palm tree and tururi sac

Each palm produces four clusters of fruit per year, of which about 6-7 kg are fruit. Harvesting is done seasonally from December to February. The material is collected in the lowlands of the Amazon rainforest and the extraction is done manually by the traditional population who lives nearby and who is familiarized with the natural difficulties of the place.

Tururi, the sac that surrounds the fruits of the Ubuçu palm tree, is constantly used by the population and artisans in the region. Moreover, the trunk, leaves, and fruits are also utilized 
by these communities from the Amazon region, in multiple ways and with the most varied types of applications (Monteiro and Baruque-Ramos 2016, 128).

The 80 raw materials were extracted from the Marajó archipelago in the state of Pará - Brazil by local communities that traditionally work with the material. In this work, we repeated the procedure of preparation of the material adopted by the Amazonian traditional population. We tried to achieve a scientific parameter respecting the traditional work. Thus, we decided not to use chemical products or other processes and treatments that can influence the properties of the material itself. To prepare the fibrous material for resin treatment for the development of composites, the steps below need to be followed as described. Nevertheless, it is important to emphasize that our intention was to replicate traditional methods in a scientific environment.

The process of development of a composite begins with the preparation of the fibrous material tururi (Figure 2). First, the weight of each specimen was measured using a Shimadzu model Ux6200H semi-analytical scale. The length was measured using a measuring tape.

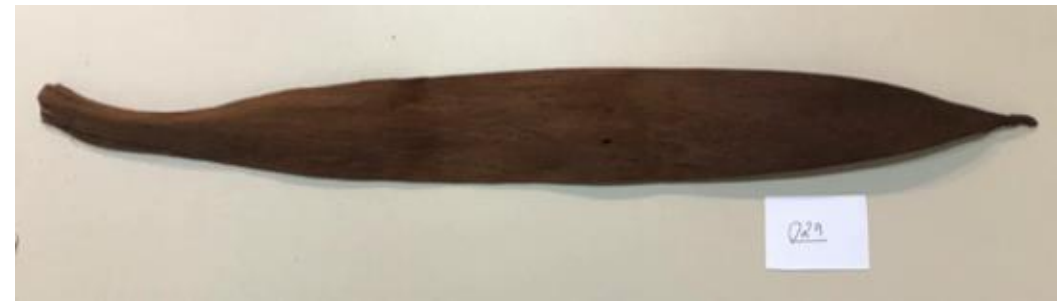

Figure 2: Tururi sac in the beginning of the process

The second step is to measure the thickness of the samples. Due to the irregular aspect of the material, we decided it was necessary to measure the sample in six different points (Figure 3). The Digital External Micrometer was used with a measurement in the range from 0 to $25 \mathrm{~mm}$ (Mitutoyo, model MC-004) (Figure 4).

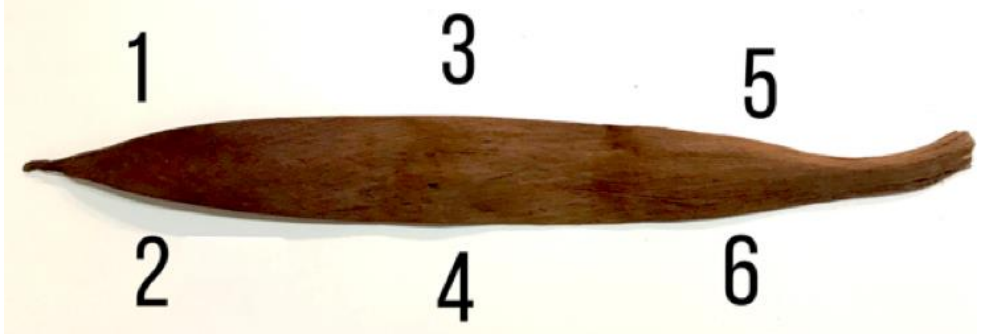

Figure 3: The measurement point scheme

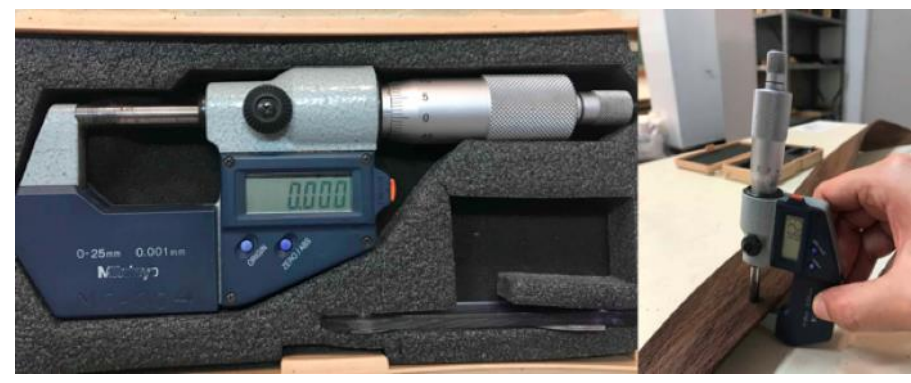

Figure 4: The Digital External Micrometer

After the measurements, a photograph was taken of each sample. At this point, the cleaning procedures adopted by the traditional Amazonian population were ready to begin. First, the tururi bag was immersed in water for 24 hours. 
Then, after a visual analysis, the sac was open with scissors. We decided where to cut aiming for the preservation of the sac, choosing to cut the most damaged parts. Figure 5 shows the two options. The priority was to keep the side with minor damage. For this sample, side (a) was chosen, as there were some visible broken spots on side (b).

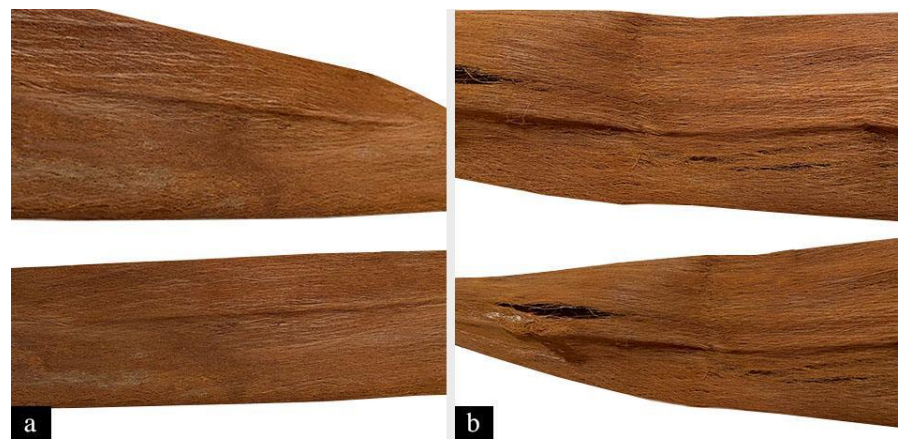

Figure 5: Cutting scheme

The next step was cleaning the opened sac. To avoid disturbing the fiber orientation, the samples were manually brushed with a delicate bristle brush to remove seeds, impurities, and foreign matter (Figure 6). This process is important to improve the Tururi fiber surface adhesion with the resin in a composite use.

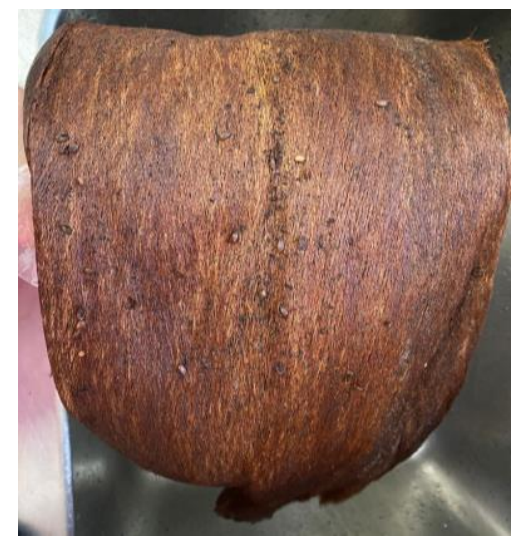

Figure 6: Seeds in the open tururi sac

The samples were then weighed while still wet and then inserted in the greenhouse with circulation and air renewal (Marconi, model MA 035) (Figure 7). As a drying criterion, the temperature was set at $45{ }^{\circ} \mathrm{C}$, with the first measurement of the sample mass after 24 hours and the second measurement 6 hours later. The value of $1 \%$ difference between the two masses was then defined as the stopping criterion in the 6 hours difference between the two weight measurements.

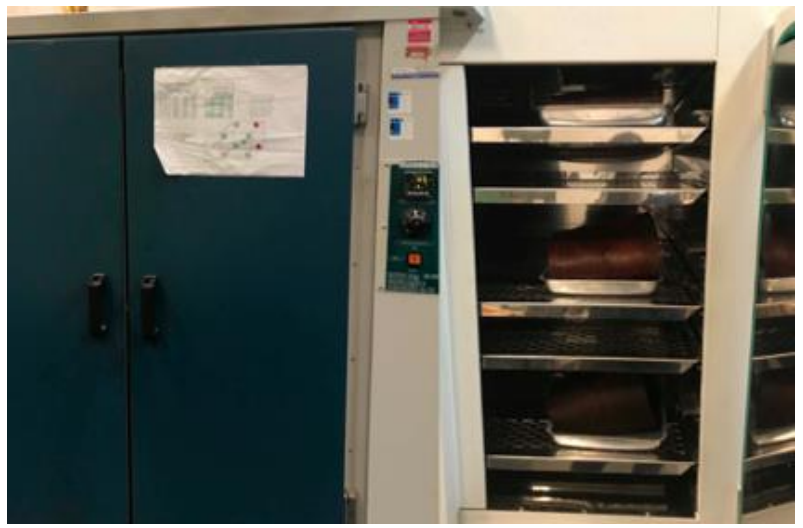

Figure 7: Drying process 
The final step was to cut the washed and dried sacs. Both ends need to be cut to convert them into a single layer of rectangular shape with a dimension of $30 \mathrm{~cm} \times 15 \mathrm{~cm}$. The leftover material can be used as well, therefore we decided to cut them into strips, taking advantage of the irregular shape of the surplus. Figure 8 shows the final sample and the strips made from the surplus (Seyam et al. 2017, 19).

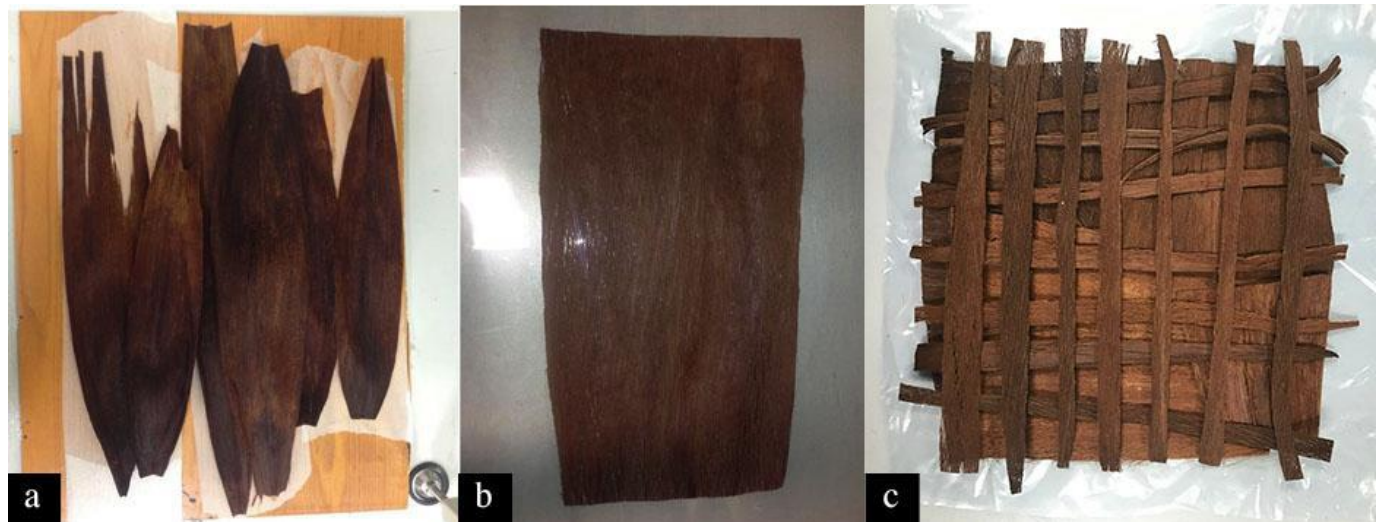

Figure 8: (a) Washed tururi; (b) a sac after trimming and cutting to form single layer preform; (c) and strips made from the surplus of the material

It is important to mention that, in order to achieve a cleaner process in terms of sustainability, it was defined that, for every three samples, 8 liters of water would be used. After the cleaning process, the impurities in the water were filtered and then the disposal was made.

\section{Discussion}

Here are presented the results of the cleaning process of the tururi fiber (the natural sac). Those pieces of information are valuable for both the application of the material as a design component as well as the characterization of the material itself. In total, 80 samples were measured, cleaned, and dried.

Regarding the measurement of the samples, the average length was $88.45 \mathrm{~cm}$, with a standard deviation of $8.85 \mathrm{~cm}$ and a variation coefficient of $10 \%$. It is important to notice that, after being cut in the desired piece, the material results in big amounts of remnants, due to the long length of the sac.

Concerning the material's weight, the results show an average value of $32.40 \mathrm{~g}$, with a standard deviation of $7.57 \mathrm{~g}$ and a variation coefficient of $23.38 \%$. It was noticed that this material is lightweight and such information has to be taken into account in a design project. In reference to the thickness, the results obtained for 80 samples are shown below (Table 1).

\begin{tabular}{cccc}
\hline Point & Average $(\mathbf{m m})$ & $\begin{array}{c}\text { Standard deviation } \\
(\mathbf{m m})\end{array}$ & CV (\%) \\
\hline 1 & 1,84 & 0,41 & 22,51 \\
2 & 1,81 & 0,42 & 23,41 \\
3 & 1,35 & 0,32 & 23,73 \\
4 & 1,33 & 0,30 & 22,97 \\
5 & 1,31 & 0,25 & 19,20 \\
6 & 1,30 & 0,28 & 21,56 \\
\hline
\end{tabular}

Table 1: Thickness measurement results

\section{Conclusions}

As expected for a natural material, we noticed the results regarding its measurement had variations in all 80 samples. Therefore, we emphasize the importance of defining the size of the sample and to prepare a cutting scheme. As an example, here we used the $30 \times 15 \mathrm{~cm}$ 
predefined size, which also collaborated with the organization and usage of the surplus material. In such matters, every part of the tururi sacs is exploited, as proposed by the Brazilian Amazonian traditional population in its original use.

With reference to the measurement, cleaning, and drying process carried out with the samples, our process shows that, with a reduced number of steps and using a few laboratory equipment, it is possible to perform the preparation of the material. Such information is important because, if followed as demonstrated, the academic community can replicate the steps in different contexts, even if appropriate laboratories are unavailable.

Additionally, the process may stimulate the search for other materials that are underutilized until this day, proposing a curious investigation, led by creative goals, that can change the actual production of products. For the design field, it is a valuable path, and we hope that it can lead to the development of new materials and technologies, conducted creatively by designers.

\section{References}

Ashby, M., and K. Johnson. 2011. Materiais e Design: Arte e ciência da seleção de materiais no design de produto. Translated by A. S. Marques. Rio de Janeiro: Elsevier.

Ayala-Garcia, C., and V. Rognoli. 2017. "The new aesthetic of DIY-materials". Design Journal 20: S375-S89. https://doi.org/10.1080/14606925.2017.1352905.

Karana, E., P. Hekkert, and P. Kandachar. 2008. "Material considerations in product design: A survey on crucial material aspects used by product designers". Materials \& Design 29, no. 6: 1081-89. https://doi.org/10.1016/j.matdes.2007.06.002.

Manzini, E. 1993. A matéria da invenção. Translated by P. A. Dias. Porto: Porto Editora.

Manzini, E., and C. Vezzoli. 2008. O desenvolvimento de produtos sustentáveis. Translated by A. de Carvalho. São Paulo: EdUSP.

Monteiro, A., and J. Baruque-Ramos. 2015. "Amazonian tururi palm fiber material (Manicaria saccifera Gaertn)". In Natural Fibres: Advances in science and technology towards industrial applications, edited by R. Fangueiro and S. Rana, 127-37. Germany: RILEM Bookseries.

Seyam, A. F. M., A. S. Monteiro, M. Midani, and J. Baruque-Ramos. 2017. "Effect of structural parameters on the tensile properties of multilayer 3D composites from Tururi palm tree (Manicaria saccifera Gaertn) fibrous material". Composites Part B-Engineering 111: 17-26. https://doi.org/10.1016/j.compositesb.2016.11.040.

Rognoli, V., M. Bianchini, S. Maffei, and E. Karana. 2015. "DIY materials". Materials \& Design 86: 692-702. https://doi.org/10.1016/j.matdes.2015.07.020.

\section{Acknowledgments}

The authors would like to acknowledge the Institute for Technological Research (IPT) and its foundation (FIPT) for the financial and institutional support, through the New Talents Program. 